\title{
Statistical properties of exoplanets
}

\section{The period distribution: Constraints for the migration scenario}

\author{
S. Udry, M. Mayor, and N. C. Santos
}

Observatoire de Genève, 51 Ch. des Maillettes, 1290 Sauverny, Switzerland

Received 4 November 2002 / Accepted 16 May 2003

\begin{abstract}
Interesting emerging observational properties of the period-mass distribution of extra-solar planets are discussed. New recent detections confirm the already emphasized lack of massive planets $\left(m_{2} \sin i \geq 2 M_{\mathrm{Jup}}\right)$ on short-period orbits $(P \leq$ 100 days). Furthermore, we point out i) a shortage of planets in the 10-100 day period range as well as ii) a lack of light planets $\left(m_{2} \sin i \leq 0.75 M_{\text {Jup }}\right.$ ) on orbits with periods larger than $\sim 100$ days. The latter feature is shown not to be due to smallnumber statistics with Monte-Carlo simulations. These observational period-related characteristics are discussed in the context of the migration process of exoplanets. They are found to be in agreement with recent simulations of planet interactions with viscous disks. The observed valley at a few tens of days in the period distribution is interpreted as a transition region between two categories of planets that suffered different migration scenarios. The lack of light planets on longer-period orbits and the corresponding intriguing sharp limit in mass is tentatively explained by the runaway migration process recently studied by Masset \& Papaloizou (2003). The observed properties also have implications for the observation strategies of the on-going surveys and of future higher-precision searches.
\end{abstract}

Key words. techniques: radial velocities - stars: planetary systems

\section{Introduction}

The most remarkable feature of the sample of known extrasolar planets is undoubtedly the variety of their orbital characteristics, which challenges the conventional views of planetary formation. Amongst the most peculiar candidates are the giant planets orbiting very close to their parent stars, in contrast to the prediction of the standard model (e.g. Pollack et al. 1996) that they formed first from ice grains ${ }^{1}$ in the outer region of the system where the temperature of the stellar nebula is not too high. The accommodation of this scenario to the present observations requires that the planets undergo a subsequent migration process bringing them close to the central star (see e.g. Lin et al. 1996; Ward 1997). Alternative points of view invoke in situ formation (Wuchterl 2000a,b), possibly triggered through disk instabilities (Boss 2001, 2002). Note however that, even in such cases, subsequent disk-planet interactions leading to migration are expected to take place as soon as the planet is formed.

The number of known extra-solar planets exceeding 100 , a statistically significant sample is now available from which we can determine meaningful distributions of planetary characteristics (Udry et al. 2003a; Marcy et al. 2003), and so try

Send offprint requests to: S. Udry, e-mail: stephane.udry@obs.unige.ch

${ }^{1}$ Such grain growth provides the supposed requisite solid core around which gas could rapidly accrete, over the lifetime of the protoplanetary disk $\left(\sim 10^{7} \mathrm{y}\right)$. to point out useful constraints for the planet-building models and then possibly discriminate between the different proposed scenarios.

In a new series of papers, we will try to emphasize the emerging properties of planet-host stars and characteristics of the different orbital-element distributions of exoplanetary systems and discuss their implications for our understanding of planetary formation and evolution. This paper (Paper I) will be dedicated to the period/separation distribution of exoplanets. Sharp features in the period versus mass diagram start to emerge and provide strong observational constraints for the migration scenario. More specifically, we re-discuss the lack of massive planets on short-period orbits recently pointed out (Udry et al. 2002; Zucker \& Mazeh 2002; Pätzold \& Rauer 2002). We also emphasize the clear emergence of a planet shortage in the 10-100d period range (a period valley) and we point out a sharp lower mass limit for the detected planets on "longer"-period orbits $(P \geq 100 \mathrm{~d})$. The later part of the paper will be dedicated to a discussion of the proposed explanations of these important observational findings and the possible implications for the future surveys.

A second paper in this series (Santos et al. 2003, Paper II) deals with the metallicity of the stars harbouring planets, providing accurate spectroscopic parameters for the most recent candidates, confirming known global properties and also pointing out new emerging features. In a third paper, Eggenberger et al. (2003, Paper III) examine the effect of binarity on planet 


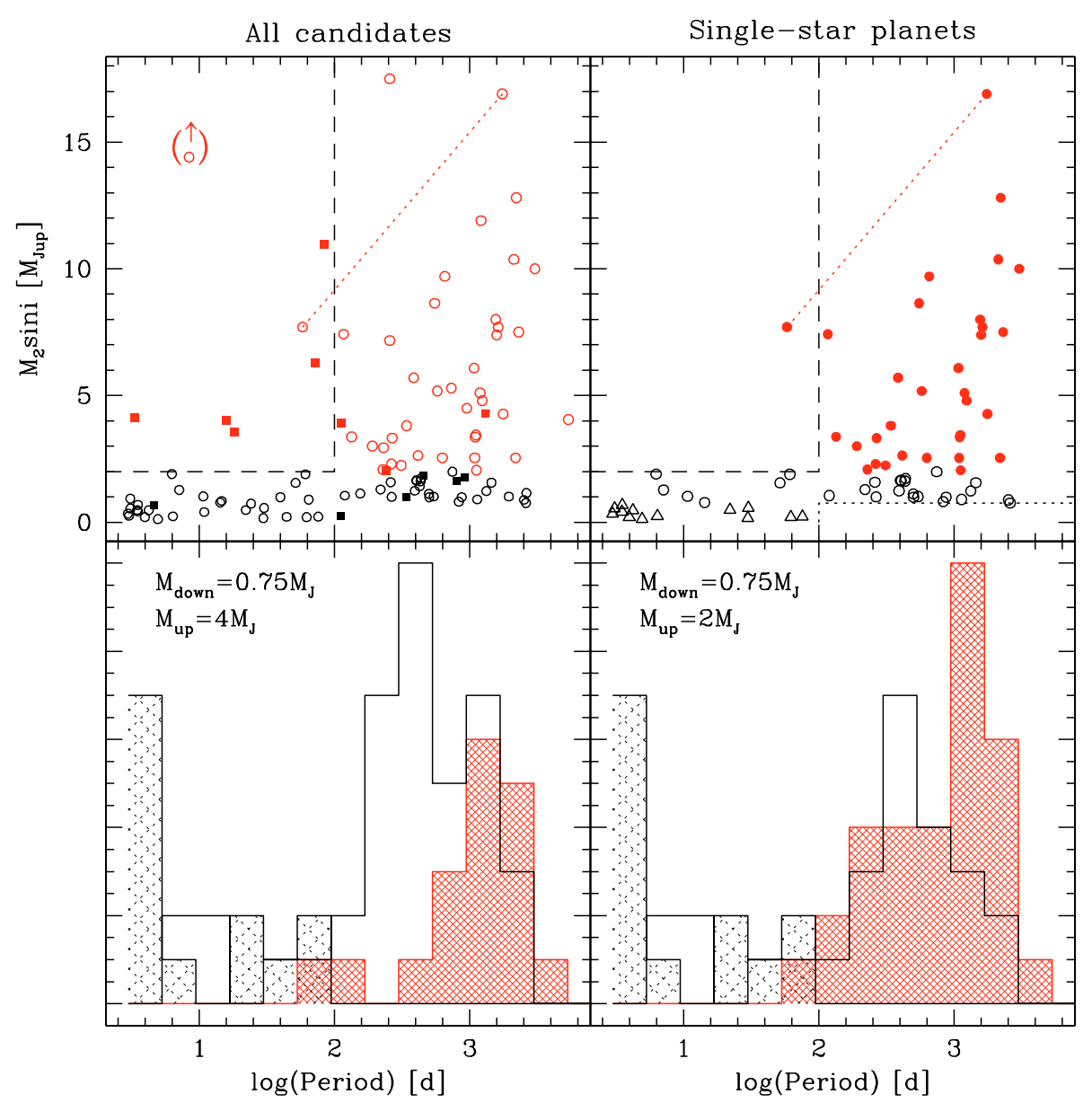

Fig. 1. Upper panels: Minimum masses versus periods for known exoplanet candidates. In the left panel, filled squares indicate planets in binaries (Paper III; Udry et al. 2003b,c) whereas circles are used for planets around single stars. The point in "()" represents HD 162020 for which synchronisation arguments indicate a probable mass in the brown-dwarf regime (Udry et al. 2002). In the right panel, only planets orbiting single dwarf stars are represented (i.e. planets in binaries and planets orbiting evolved stars have been discarded). A different coding is used for massive ( $m_{2} \sin i \geq 2 M_{\mathrm{Jup}}$; filled symbols), intermediate-mass ( $m_{2} \sin i$ between 0.75 and $2 M_{\mathrm{Jup}}$; open circles), and lighter $\left(m_{2} \sin i \leq 0.75 M_{\mathrm{Jup}}\right.$; open triangles) candidates. The components of the possible multi brown-dwarf system HD 168443 are linked by a dotted line. The dashed and dotted lines in the panels indicate limits at $P=100 \mathrm{~d}$ (vertical), at $m_{2} \sin i=2 M_{\text {Jup }}$ (horizontal left), or at $m_{2} \sin i=0.75 M_{\text {Jup }}$ (horizontal right). Lower panels: Period distributions of the planets orbiting single dwarf stars (shown in the upper-right panel) for different mass regimes: light-shaded histograms are for the lightest planets $\left(m_{2} \sin i \leq M_{\text {down }}=0.75 M_{\mathrm{Jup}}\right)$, open histograms are for intermediate masses (between $M_{\text {down }}$ and $M_{\text {up }}$-right: $M_{\text {up }}=2 M_{\text {Jup }}$; left: $\left.M_{\text {up }}=4 M_{\text {Jup }}\right)$, whereas the dark-shaded histograms are for the more massive candidates $\left(m_{2} \sin i>M_{\text {up }}\right)$.

formation and evolution. The presence of a close stellar companion is found to bring more massive planets close-in, in agreement with simulations by Kley (2001).

\section{Observational emerging properties}

\subsection{No massive planets on short-period orbits}

In a recent discussion of the statistical properties of massive planets versus lighter ones (Udry et al. 2002), we emphasized a shortage of massive planets on short-period orbits, based on the sample of about 80 exoplanets known at that time. This feature was also simultaneously pointed out by Zucker \& Mazeh (2002). These authors furthermore verified its statistical significance and very interestingly examined the possible influence of binarity on the mass-period relation of exoplanets, an indication of potential different formation and evolution processes for planets in binaries and planets around single stars.
With now more than 100 very low-mass candidates (with $m_{2} \sin i \leq 18 M_{\mathrm{Jup}}$ ), the lack of short-period massive planetary companions becomes even clearer, as seen in Fig. 1. When we neglect the multiple-star systems ${ }^{2}$ (upper-right panel) in which the planetary formation or evolution could follow different paths (Zucker \& Mazeh 2002; Udry et al. 2003b,c, Paper III), a complete void of candidates is observed in the diagram for masses larger than $\sim 2 M_{\text {Jup }}$ and periods smaller than $\sim 100$ days $^{3}$. The only remaining point is HD $168443 \mathrm{~b}$, member of a possible multi brown-dwarf system (Marcy et al. 2001; Udry et al. 2002).

\footnotetext{
2 As determined by dedicated adaptive optics programmes or unveiled by previous spectroscopic measurements (see Paper III; Udry et al. 2003b,c, for a review).

3 The 4 known candidates of planets orbiting an evolved star have also been discarded. They however have no influence on the results of this study.
} 
Several processes have been proposed to explain the lack of massive planets on short-period orbits. In the context of the migration scenario, they mainly follow two different approaches: i) type II migration (after a gap opens in the disk) is shown to be less effective for massive planets i.e. massive planets stay farther out than lighter ones, or ii) when the planet reaches the central regions, some process related to planet-star interactions provokes mass transfer from the planet to the star - decreasing the mass of the former - or leads massive planets to fall into the central star. These approaches will be discussed in more detail in Sect. 3.

The possibility that multi-planet chaotic interactions send the lighter candidates in the inner regions (or out) of the system whereas the massive ones stay in the outer part may also be invoked (Rasio \& Ford 1996; Weidenschilling \& Marzari 1996). The frequency of planets ending very close to the central star seems however to be small (Ford et al. 2003).

\subsection{A period valley between 10 and 100 -d periods}

In the lower panels of Fig. 1 and upper panel of Fig. 2, another very interesting feature is coming out of the period/separation distribution. We observe a shortage of planets with periods between roughly 10 and 100 days. This observational property is mainly due to the light candidates $\left(m_{2} \sin i \leq 2 M_{\mathrm{Jup}}\right)$. As seen in the previous section, massive planets orbiting single stars are almost exclusively found on longer-period orbits. They probably form and stay further out (see discussion in Sect. 3). On the other hand, lighter planets are found at all distances from their star ${ }^{4}$. This mass-dependent behaviour is also clearly illustrated by the cumulative functions presented in the lower panel of Fig. 2 for two planetary mass regimes.

The features appearing in the planetary period distribution - peak at short periods and rise at intermediate periods - seem significant and not due to observational biases. The peak at short period is formed by the pile-up of migrating planets, stopped close to the central star. As seen in Fig. 1, it is almost exclusively composed of the lowest mass planets $\left(m_{2} \sin i \leq 0.75 M_{\mathrm{Jup}}\right)$ and not due to observational biases as the detection limit at $10 \mathrm{~ms}^{-1}$ is at $P \simeq 4000$ days for $m_{2} \sin i=0.75 M_{\text {Jup }}$ (with $M_{1}=1 M_{\odot}$ and $e=0$. The effect of Jupiter on the Sun is $12 \mathrm{~ms}^{-1}$ ). The rise of the distribution at longer periods is now emerging thanks to the increase of the timebase of the radial-velocity surveys. More and more longperiod planets are being detected. This rise is significant in the sense that, for a given mass range, similar planets on shorterperiod orbits would have been easier to detect than the actual ones. The observed valley in the period distribution can then just be seen as a transition region between two categories of planets which suffered different migration behaviours.

\subsection{A sharp mass transition in the migration process?}

A third important feature emerging from the massperiod diagram is the apparent lack of very light planets

\footnotetext{
4 except the lightest ones $\left(m_{2} \sin i \leq \sim 0.75 M_{\text {Jup }}\right)$ at periods longer than $\sim 100$ days (see next section).
}

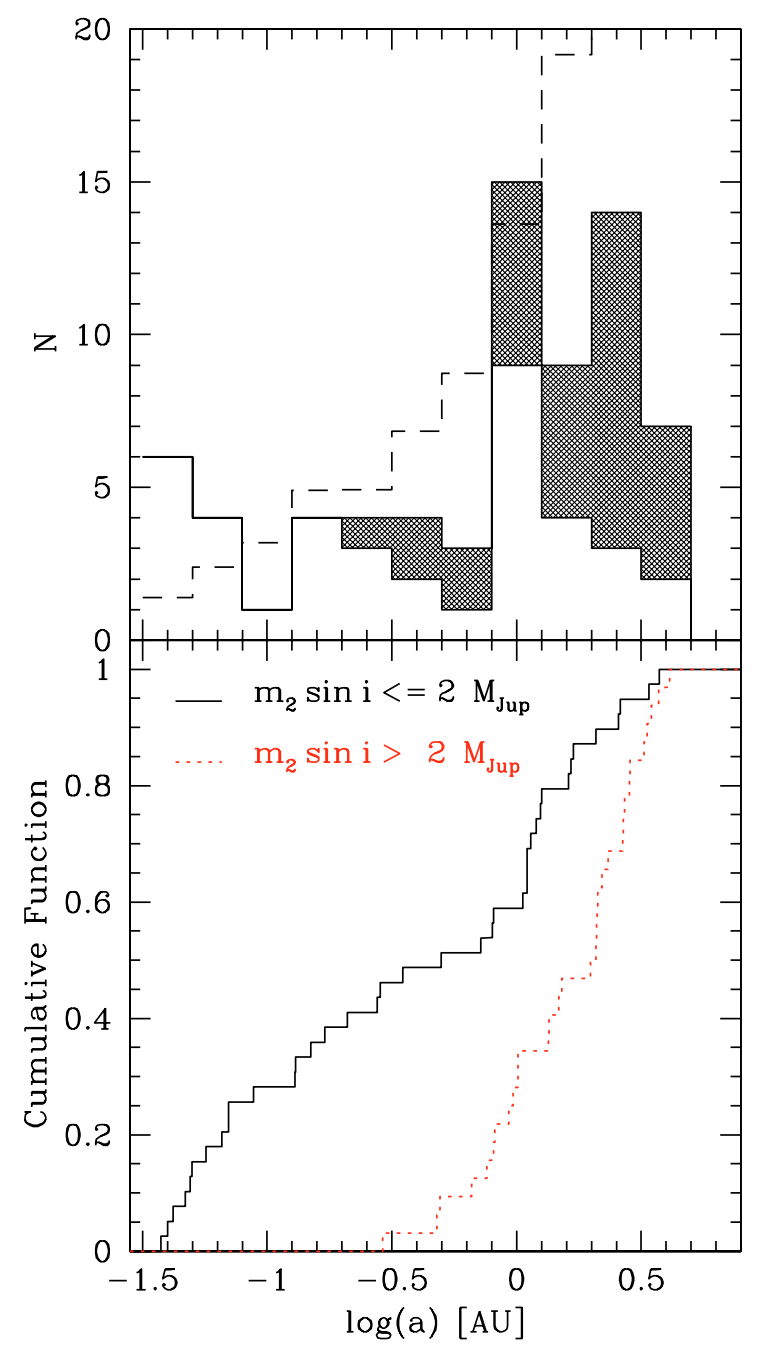

Fig. 2. Upper panel: Distribution of separations of the known exoplanets orbiting single dwarf stars (log scale) compared to the similar distribution of the Trilling et al. (2002) simulation end-states (dashed line). The planets more massive than $2 M_{\text {Jup }}$ are indicated by the shaded part of the histogram. The planet shortage in the $0.06 \leq a \leq 0.6 \mathrm{AU}$ separation range (10-100 d) comes out very clearly. Lower panel: Cumulative functions of $\log a$ for the 2 mass regimes (limit at $2 M_{\text {Jup }}$ ). The preponderance of light planets close-in and the location of massive planets further out is clearly emphasized. The Kolmogorov-Smirnov probability for the 2 distributions to come from the same population is $6.7 \times 10^{-4}$.

( $m_{2} \sin i \leq 0.75 M_{\text {Jup }}$ ) with periods larger than $\sim 100$ days. This feature is already visible in the upper-right diagram of Fig. 1 (limited by the dotted line). It becomes obvious when the planetary minimum mass is displayed with a log scale (Fig. 3).

Of course this feature could be related to the observational bias inherent to the radial-velocity technique for planet search that makes the detection more difficult for distant and/or lighter planets. To obtain a quantitative idea of the effect of this bias, we have also plotted in Fig. 3 lines indicating the locations of the radial-velocity signals (semi-amplitude $K$ of 30,10 and $3 \mathrm{~ms}^{-1}$ ), expected on a solar-mass star due to planets on circular 


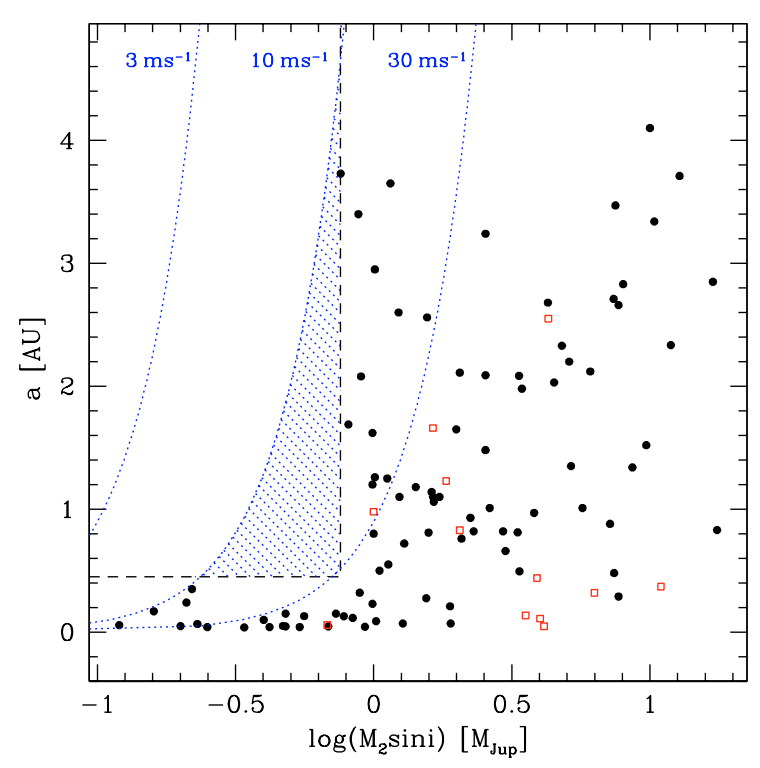

Fig. 3. Mass-separation diagram for the known exoplanet candidates. The dotted lines illustrate the radial-velocity semi-amplitude $(3,10$ and $30 \mathrm{~ms}^{-1}$ ) expected on a solar-mass star due to planets on circular orbits with given minimum masses and separations. The shaded area empty of planets is shown not to be due to small number statistics (see text). Planets in binaries are indicated by open symbols.

orbits with given minimum masses and semi-major axes ${ }^{5}$. The line for $K=10 \mathrm{~ms}^{-1}$ gives the approximate $3 \sigma$-limit of the present most precise surveys reaching precisions of $\sim 3 \mathrm{~ms}^{-1}$ (e.g. Vogt et al. 2000; Queloz et al. 2001). Some known candidates have been found very close to this limit, at low mass and small distance or at high mass and large distance ${ }^{6}$. However, we clear see an "intermediate" region (hatched area), delimited by $m_{2} \sin i=0.75 M_{\mathrm{Jup}}, P=100 \mathrm{~d}$ and $K=10 \mathrm{~ms}^{-1}$, where no planetary candidates have been found. A striking feature of this area is its very sharp limit in mass.

Is this empty region just due to the mentioned observational bias and the small-number statistics? We have in particular to worry about the observed effect coming from the shortage of candidates with periods in the 10-100 day range, as pointed out in the previous section. So, to check the statistical significance of the emptiness of the hatched area in the figure (i.e. to see whether it mainly comes from the small number of known planets), we performed Monte-Carlo simulations. The idea is to obtain $10^{6}$ realizations of the diagram, reproducing the actual observational biases of the known sample, and estimate from them the probability to have no point in the considered zone.

There are basically two ways of producing samples resembling to the observations: either i) take realistic planetary parameter distributions and model the observational biases or ii) draw the test samples from the actually observed distributions in which the acting biases are built in. As the "real"

\footnotetext{
${ }^{5}$ The primary mass and eccentricity effects are not expected to be important as the radial-velocity semi-amplitude scales with (1 $\left.e^{2}\right)^{-1 / 2} M_{1}^{-2 / 3}$.

${ }^{6}$ We can also note that these candidates "close" to the detection limit do not present special values of the eccentricity and primary or planetary masses.
}

distributions are not known a priori, we have adopted the second approach:

i) We randomly select the orbital eccentricities, the primary masses, the planet minimum masses and separations from the actual observed distributions of $e, M_{1}, m_{2} \sin i$ and $\log a$ of the detected exoplanets. This ensures in particular that we are taking into account the effect of the orbital eccentricity and of the stellar mass on planet detection. It also produces distributions that reproduce the observed brown-dwarf desert and the period valley.

ii) Since the distributions of separations are different for light and massive planets (Fig. 1), we do the selection for two mass regimes independently (limit at $4 M_{\text {Jup }}$ ).

iii) We only use the distributions for planets orbiting single dwarf stars as 1) planets in binaries may have different characteristics (Zucker \& Mazeh 2002, Paper III) and 2) the post main-sequence stellar evolution may drastically change the fate of short-period planets.

iv) We reject the events giving a radial-velocity semiamplitude smaller than $K_{\lim }=10 \mathrm{~ms}^{-1}$. They would hardly be detected by the present surveys. A less restrictive limit a $15 \mathrm{~ms}^{-1}$ is also considered to check the sensitivity of the results on the detection limit.

v) Each realization consists of a selection of 86 pseudoplanets, corresponding to the actual observations (64 "light" and 22 "massive" planets orbiting single dwarf stars, as on February 2003). For each of them we count the number $n$ of points in the "test zone". Note that the detection limit at $K_{\lim }$ is no more as well defined as in Fig. 3 but it now takes into account the orbital eccentricity and primary-mass values.

The normalized histogram of the distribution of the number of points $n$ in the test zone from our $10^{6}$ realizations is shown in Fig. 4. The probability of having no detection is only 0.00028 . A bootstrap of the procedure with 100 times $10^{5}$ realizations gives a value of $0.00025 \pm 0.00006$ (Fig. 5), in agreement with the previous result. Relaxing the detection limit to $15 \mathrm{~ms}^{-1}$ yields a probability of 0.004 . It shows the low sensitivity of the result on the chosen radial-velocity detection limit. We thus can conclude that the considered area is indeed empty of planets with a confidence level of about $99.97 \%$.

This conclusion is only valid if the observational biases are correctly taken into account. Biases related to planetary parameter distributions and detection technique are by construction included in the simulations. However, other aspects play a role. For example activity-induced radial-velocity jitter may screen planet detection. Such an effect can be neglected if we suppose that the $m_{2} \sin i, e, M_{1}$ and $a$ parameters are not correlated with activity. Another concern relates to the difficulty encountered when trying to actually derive the values for the orbital elements, what is different from just detecting radialvelocity variability. Observation timing and phase coverage are then also important parameters. These remarks emphasize the caution needed when considering the above described results. We should see them more like a trend emerging from the data rather than a real proof.

In the next section, we will propose a tentative explanation for this feature, based on recent results on runaway migration studied by Masset \& Papaloizou (2003). We will also discuss 


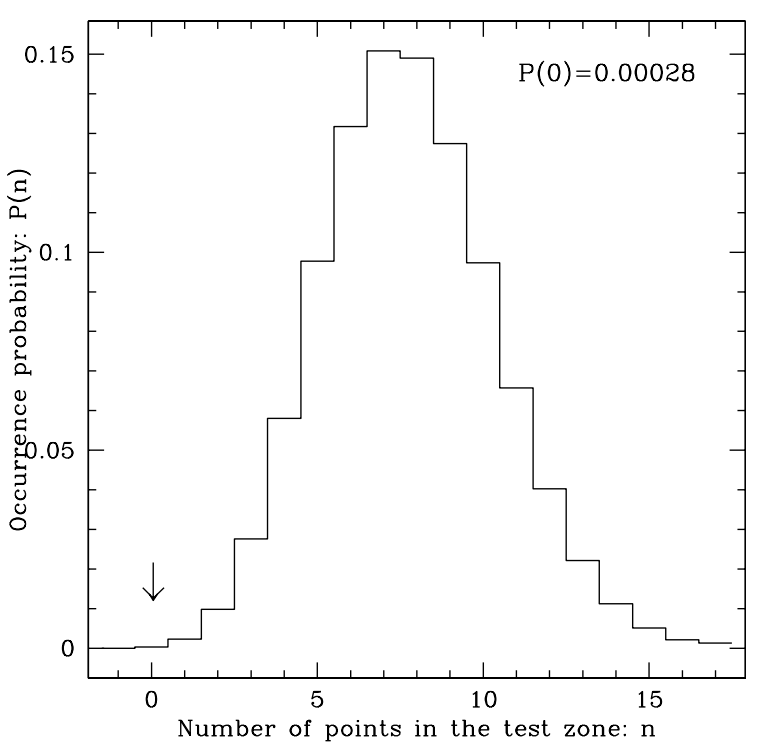

Fig. 4. Result on the statistical significance of the hatched zone in Fig. 3 based on $10^{6}$ realizations of the diagram with Monte-Carlo simulations. The figure presents the normalized histogram of the distribution of the number of points, $n$, found in the test region i.e. the occurrence probability of $n$. The arrow location at $n=0$ indicates the position of the actual observational result. Its probability of occurrence only by chance is $P(0)=0.00028$.

in the last section of this paper the implications on the radialvelocity planet-search surveys of the paucity of light planets with intermediate periods.

\section{Checking the theoretical approaches}

The different features pointed out above in the various presentations of the period/separation-mass diagram will allow us to check the processes proposed to explain the observed distribution of periods for extra-solar planet candidates.

\subsection{Low migration rate for massive planets}

A first approach to explain the lack of massive planets on shortperiod orbits invokes a low migration efficiency for higher mass planets. This view is supported by recent simulations of singleplanet migration. When the mass of the planet becomes of the order of a significant fraction of the characteristic disc mass with which it interacts, the inertia of the planet becomes important and slows down the orbital evolution (e.g. Trilling et al. 1998; Nelson et al. 2000). It also takes more time to open a gap in the disk for massive planets - eventually longer than the lifetime of the disk - and initiate type II migration (Trilling et al. 2002).

Using a model assuming a simple impulse approximation for the type II migration, no ad hoc stopping mechanism in the center and neglecting type I migration ${ }^{7}$,

\footnotetext{
7 The extremely short timescale of the type I migration represents a serious theoretical problem for planet formation. However, suggestions have been recently made that co-orbital corotation torque in thin viscous disks may decrease its dramatic effect (Artymowicz 2003) and even stop or revert it for a large set of conditions (Masset 2002). This has however to be further examined.
}

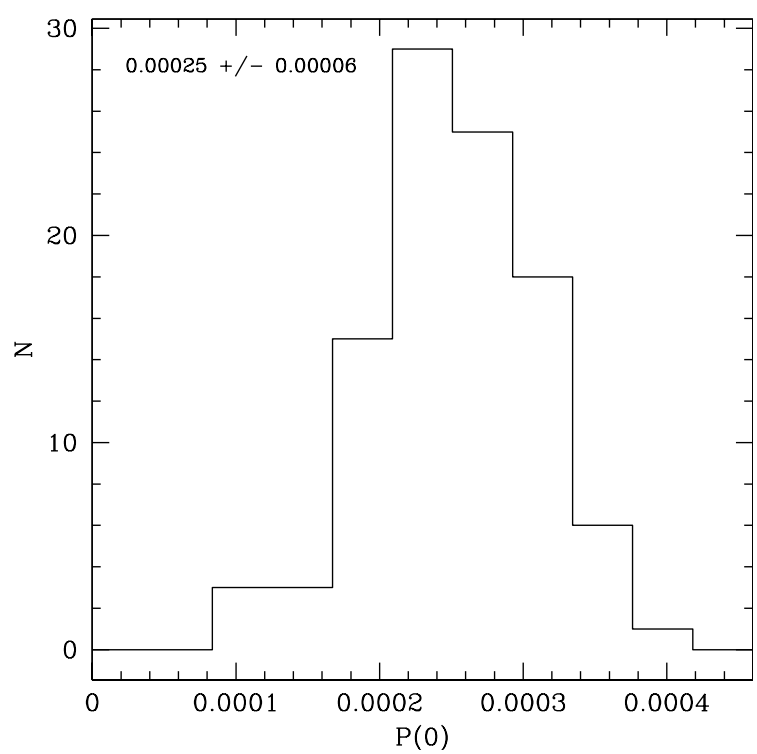

Fig. 5. Distribution of the $P(0)$ probabilities of 100 bootstrap simulations (but with $10^{5}$ realizations each) conducted to estimate the uncertainty on the value obtained in Fig. 4. They yields a value $0.00025 \pm 0.00006$ for $P(0)$.

Trilling et al. (2002) show that, for identical initial disk conditions, close-in surviving pseudo-planets have smaller masses and distant surviving pseudo-planets have larger masses. They also find that planets forming farther out migrate less rapidly and that higher-mass planets migrate on longer timescales. Thus, there should be more massive planets at intermediate and large semi-major axes than found close-in. On the contrary, the population of short-period planets should be dominated by smaller-mass planets, what is indeed observed:

- The upper panel in Fig. 2 presents the logarithmic distribution of exoplanet separations, superimposed to the Trilling et al. (2002) results. As already pointed out by these authors, if we take into account the observational bias penalizing longperiod planets in the radial-velocity surveys, the theoretical and observational results agree fairly well. The observed peak at small separations is not reproduced by the simulations of Trilling et al. because they have not introduced in their study any ad hoc process to stop the migration close to the central star.

- Furthermore, the lower panel in Fig. 2 giving the cumulative functions for 2 mass regimes (limit at $2 M_{\text {Jup }}$ ) clearly shows that low-mass planets form the predominant population of short-period orbits whereas more massive planets are located further out.

- Moreover, the lower panels of Fig. 1 indicates that you have to go further and further out to find more and more massive planets. The hatched histogram representing planets more massive than $4 M_{\text {Jup }}$ rises up to 1000 days (left panel) - although such massive planets should be easier to detect at smaller periods - whereas the maximum of the rise of the distribution of lighter-mass planets is around 400 days. If we decrease down to $2 M_{\text {Jup }}$ the limit between the 2 mass regimes, the mentioned features almost overlap (right panel), indicating a trend for the higher masses to be found at larger distances from 


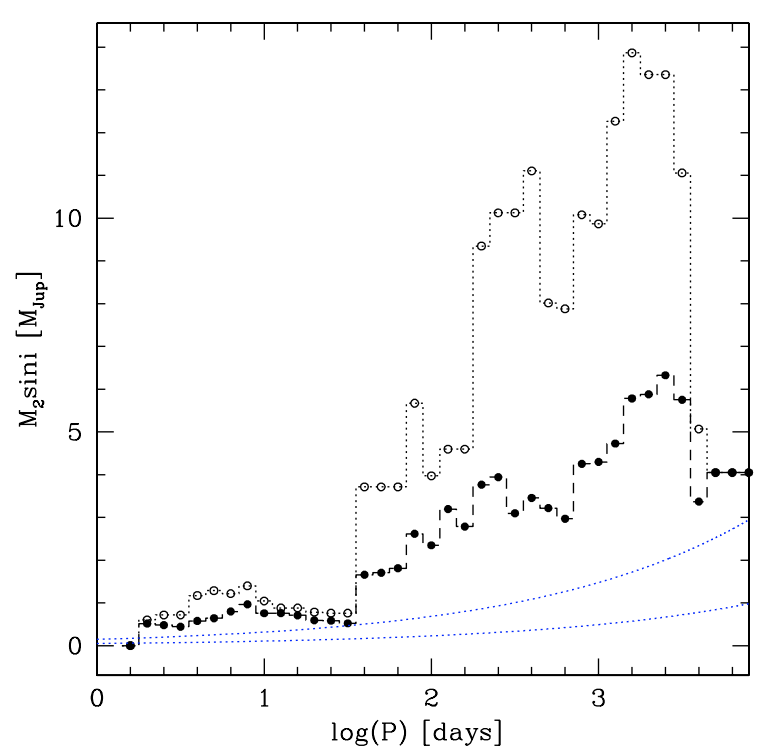

Fig. 6. Mean mass (filled circle) or highest mass (average on the 3 highest values; open circles) of planets in period smoothing windows of width $\log P$ [days] $=0.2$. A clear increase of planet maximum masses with period is observed, despite the fact that massive planets observed at a given period should be easier to detect at smaller periods. Detection limits at 10 and $30 \mathrm{~ms}^{-1}\left(M_{1}=1 M_{\odot}, e=0\right)$ are represented by the dotted lines.

the central star. This higher mass trend with period is explicited in Fig. 6 which displays the mean mass (filled circle) or higher mass (average on the 3 highest values; open circles) of planets in period smoothing windows of width $\log P[$ days $]=0.2$. The effect becomes visible around $P=40 \mathrm{~d}$ and turns to be very effective around $P=100 \mathrm{~d}$. Detection limits at 10 and $30 \mathrm{~ms}^{-1}$ are represented by the dotted lines. They show that observational biases related to the radial-velocity technique are not responsible for the observed feature. This effect is not surprising considering first that massive planets are preferentially formed in the outer regions where a larger amount of building material is available and second that higher-mass objects migrate on longer timescales. Furthermore, as the disk dissipates, the migration of more massive planets slows down earlier since their larger angular momentum produces greater resistance to diskinduced migration (Armitage et al. 2002).

\subsection{Runaway migration and lack of light planets with longer periods}

A recent study by Masset \& Papaloizou (2003) brings some insights for our understanding of the observed lack of small-mass planets $\left(m_{2} \sin i \leq 0.75 M_{\text {Jup }}\right)$ on intermediate-period orbits $(P \geq 100 \mathrm{~d})$. These authors studied the effect of co-orbital corotation torque on migrating protoplanets in the case of massive protoplanetary disks for which the planet Hill radius and the disk thickness have comparable orders of magnitude. In particular they show that, if the mass deficit created by the radial drift with the planet of the material trapped in the co-orbital region is larger than the planet mass, the migration rate undergoes a runaway which can rapidly vary the protoplanet semi-major axis by a large amount ( $50 \%$ over a few tens of orbits). The authors state that: "this can happen only if the planet mass is sufficient to create a gap in its surrounding region and if the surrounding disk mass is larger than the planet mass. This typically corresponds to planet masses in the sub-Saturnian to Jovian mass range embedded in massive protoplanetary disks".

Moreover, the simulations by Masset \& Papaloizou (2003) show that the limit in planetary mass for the appearance of the runaway regime is very steep (their Figs. 12-14). The threshold conditions for runaway may vary widely depending on the disk mass, viscosity or thickness. It however depends relatively weakly upon the planet mass on the high-mass side i.e. their is a critical mass relatively well-constrained ( $M_{\text {crit }} \simeq 1 M_{\text {Jup }}$ ) under which runaway is likely provided that the protoplanetary disk is not too light weight, and above which runaway is impossible (Masset, priv. comm.). As our observed limit at $m_{2} \sin i \simeq 0.75 M_{\text {Jup }}$ is compatible with this critical mass, there could well be a link between the statistical observed property and runaway migration.

In this picture, planets which happen to be massive enough $\left(M>M_{\text {crit }}\right)$ before reaching the central regions would end up as the scattered population on the right of Fig. 3, whereas planets that never grow enough would remain very mobile and would eventually be flushed into the very central region.

Note that another limit exist for runaway migration on the planet low-mass side. Thus, very light planets (typically much lighter than Saturn) do not encounter such type of fast migration. That can be the case for the outer icy planets of the Solar System.

\subsection{Observed constraints for migration: Summary}

In summary, putting everything together, we can try to draw a global picture for the planet migration behaviour in function of the planet mass, in single-star systems:

i) The more massive planets (typically, masses larger than $\sim 4 M_{\text {Jup }}$ ) form preferentially in the outer regions - where there is a large-enough material reservoir - and do not migrate much. None is observed within $\sim 0.5 \mathrm{AU}$ from the central star.

ii) Intermediate-mass objects migrate more easily whatever the distance where they form, the migration rate depending on the local conditions (planet mass, disk mass, viscosity, etc.). They are observed at all distances.

iii) The lighter planets (masses from sub-Saturnian to Jovian) migrate easily, possibly undergoing runaway migration bringing them in the central tenth of an $\mathrm{AU}$ from the star if the disk is massive enough. Most of them are actually found in the very central regions. None is observed with $P \geq \sim 100$ days although radial-velocity surveys are now precise enough to detect them.

iv) We know, however, from our own Solar System that this is not true for the planets that are far away or much lighter than the known exoplanets. Thus, we can speculate that for the very light planets the migration efficiency is decreasing with distance or mass. This again fits into the the runaway migration scenario, the simulations showing the existence of a transition limit in mass, on the low-mass side, for the runaway to occur. 
Unfortunately, the precision achieved by the present surveys does not allow us to determine the separation or mass limits at which the migration slows down significantly. Good hope to answer this question is however brought by the future surveys at higher precision (like HARPS at $1 \mathrm{~ms}^{-1}$, Pepe et al. 2002) that will increase our detection sensibility by a large factor (curve at $K=3 \mathrm{~ms}^{-1}$ in Fig. 3).

This observational view of planet migration may be changed by the influence of a perturbing stellar companion (Paper III) that will make the massive planets migrate inwards faster or make closer-in intermediate-mass planets grow bigger (Kley 2001).

Finally, we have to stress that although theory suggests that it is possible to form massive planets closer to the stars than the ice-boundary limit (Papaloizou \& Terquem 1999; Bodenheimer et al. 2000; Sasselov \& Lecar 2000), within the migration scenario, there is no observational requirement for planets to form at separations smaller than a few AU.

\subsection{Central star-planet interaction}

From the central star-planet interaction point of view, several scenarios were proposed to explain the lack of massive planets on short-period orbits as alternatives to the above developed arguments in the context of the migration scenario.

Pätzold \& Rauer (2002) proposed to explain the lack of massive close-in planets by tidal interactions between the planet and its central star. If the planet orbital period is smaller than the stellar rotation period, tidal friction will spin up the star whereas the semi-major axis of the planetary orbit is decreased, eventually reaching the Roche zone of the central star on a short timescale. These authors pointed out that massive planets spiral in much faster than others, explaining thus the observed trend.

Trilling et al. (1998) had already addressed this problem in also considering mass transfer between the planet and the star. Some of the pseudo-planets in their simulations reach the Roche lobe limit, start to lose mass and thus experience an outward torque from the Roche lobe overflow. They estimate the maximum mass of surviving planets to be around $2 M_{\text {Jup }}$, corresponding to what is indeed observed.

Another approach could relate to the evaporation of the planet atmosphere when the planet comes close to the central star, decreasing thus its mass. Up to now, studies based on the $T_{\text {eff }}$ of the heated close-in planets have shown that hydrogen evaporation was not efficient in the case of the known Hot Jupiters (e.g. Mayor \& Udry 2000). However, new developments based on the estimate of the exospheric temperature of HD $209458 \mathrm{~b}$, evaluated to be much higher than $T_{\text {eff }}$ (Vidal-Madjar 2003), call for a complete reconsideration of the question.

These approaches are very interesting to explain the period distribution of planets very close to the central stars ( $a \leq$ $0.1 \mathrm{AU}$, with a pile up around 3 days), especially considering that the peak at very short periods is almost entirely formed by the lightest mass planets $\left(m_{2} \sin i \leq 0.75 M_{\text {Jup }}\right)$. However, they do not explain the extended range of periods $(P \leq 100 \mathrm{~d})$ in which no planets more massive than $\sim 2 M_{\text {Jup }}$ are found. This large interval supports the non-migration of massive companions rather than the disappearance of close-in migrating massive planets.

\section{Summary and concluding remarks}

To summarise the main observational features pointed out in this paper and their main implications, we have that:

1) No massive planets $\left(m_{2} \sin i \geq \sim 2 M_{\mathrm{Jup}}\right)$ are found on short-period orbits $(P<\sim 100 \mathrm{~d})$ around single stars. This is not an observational bias as these candidates are the easiest ones to detect.

2) The maximum mass of detected planets per period interval increases with distance to the central star (Fig. 6). This is also a solid result as massive planets found at a given distance are easier to detect closer in.

3) The above points 1) and 2) suggest that the migration rate of planets decreases with increasing mass of the planetary companion. This result agrees with recent simulations of migrating planets in viscous disks (Trilling et al. 1998, 2002; Nelson et al. 2000). We thus expect a large number of massive planets to be on long-period orbits and so to be still undetected because of the limited duration of the present surveys. A large number a lower-mass planets probably also exist on long-period orbits; they are however more difficult to detect. These represent primary targets for future higher-precision surveys (like e.g. HARPS, Pepe et al. 2002) whereas the youngest among the formers are interesting targets for direct imaging of planetarytype objects. This result is also very important for on-going radial-velocity planet searches from which we now expect an increasing number of planet candidates as the survey durations will grow. The "older" the survey, the higher the expected detection rate. In this context, the first epoch measurement of the targets is an important parameter for planet detection.

4) We observe a shortage of planets with periods in the 10$100 \mathrm{~d}$ range. This valley in the distribution is located just inbetween the peak of light planets that have migrated inwards and were stopped close to the central star and the rise of the distribution due to the increasing number of detected planets with longer periods.

5) Up to now, no planet candidates with very low masses ( $m_{2} \sin i \leq 0.75 M_{\mathrm{Jup}}$ ) have been detected on orbits with periods longer than $\sim 100$ days. This trend seems significant and not due to small-number statistics or to the particular shape of the period or separation distributions. The limit is sharp in mass, indicating a strong transition in the migration process for different mass regimes. These features are in agreement with predictions of runaway migration simulations of planets lighter than $\sim 1 M_{\text {Jup }}$ in massive protoplanetary disks (Masset \& Papaloizou 2003).

Note however that this lack of light planets on intermediate periods may not be confirmed for much lighter planets or for planets in the considered mass range but formed far out from the central star (e.g. Neptune- and Uranus-type planets), that are still out of reach of the present observational facilities and detection techniques. 
In conclusion, we have pointed out a clear dependence of migration on mass and distance from the central star, providing important constraints for models of planetary formation (migration). More quantitative constraints for the different mass regimes will require a better detection capability of the surveys, to diminish the effect of the observational bias in the distributions and thus increase the confidence we can put in the derived trends, especially for the lower-mass planets.

Acknowledgements. This study has benefited from fruitful discussions with Frederic Masset and Willy Benz. We thank the Geneva University and the Swiss NSF (FNRS) for their continuous support of our planet-search projects. Support to N.S. from Fundação para a Ciência e Tecnologia (Portugal) in the form of scholarships is gratefully acknowledged.

\section{References}

Armitage, P., Livio, M., Lubow, S., \& Pringle, J. 2002, MNRAS, 334, 248

Artymowicz, P. 2003, in Scientific Frontiers in Research on Extrasolar Planets, ed. D. Deming, \& S. Seager, ASP Conf. Ser., in press

Bodenheimer, P., Hubickyj, O., \& Lissauer, J. 2000, Icarus, 143, 2

Boss, A. 2001, ApJ, 563, 367

Boss, A. P. 2002, ApJ, 576, 462

Eggenberger, A., Udry, S., \& Mayor, M. 2003, A\&A, submitted (Paper III)

Ford, E., Rasio, F., \& Yu, K. 2003, in Scientific Frontiers in Research on Extrasolar Planets, ed. D. Deming, \& S. Seager, ASP Conf. Ser., in press

Kley, W. 2001, in The Formation of Binary Stars, ed. H. Zinnecker, \& R. Mathieu, IAU Symp., 200, 511

Lin, D., Bodenheimer, P., \& Richardson, D. 1996, Nature, 380, 606

Marcy, G., Butler, R., Vogt, S., et al. 2001, ApJ, 555, 418

Marcy, et al. 2003, in Scientific Frontiers in Research on Extrasolar Planets, ed. D. Deming, \& S. Seager, ASP Conf. Ser., in press

Masset, F. 2002, A\&A, 387, 605

Masset, F., \& Papaloizou, J. 2003, ApJ, 588, 494
Mayor, M., \& Udry, S. 2000, in Disks, Planetesimals and Planets, ed. F. Garzón, C. Eiroa, D. de Winter, \& T. Mahoney, ASP Conf. Ser., 219, 441

Nelson, R., Papaloizou, J., Masset, F., \& Kley, W. 2000, MNRAS, 318,18

Pätzold, M., \& Rauer, H. 2002, ApJ, 568, L117

Papaloizou, J., \& Terquem, C. 1999, ApJ, 521, 823

Pepe, F., Mayor, M., Rupprecht, G., et al. 2002, The Messenger, 110, 9 Pollack, J., Hubickyj, O., Bodenheimer, P., et al. 1996, Icarus, 124, 62 Queloz, D., Mayor, M., Udry, S., et al. 2001, The Messenger, 105, 1

Rasio, F., \& Ford, E. 1996, Science, 274, 954

Santos, N. C., Israelian, G., Mayor, M., Rebolo, R., \& Udry, S. 2003, A\&A, 398, 363

Sasselov, D., \& Lecar, M. 2000, ApJ, 528, 995

Trilling, D., Benz, W., Guillot, T., et al. 1998, ApJ, 500, 428

Trilling, D., Lunine, J., \& Benz, W. 2002, A\&A, 394, 241

Udry, S., Mayor, M., Naef, D., et al. 2002, A\&A, 390, 267

Udry, S., Mayor, M., \& Queloz, D. 2003a, in Scientific Frontiers in Research on Extrasolar Planets, ed. D. Deming, \& S. Seager, ASP Conf. Ser., in press

Udry, S., Eggenberger, A., \& Mayor, M. 2003b, in Planetary systems and planets in systems, ed. S. Udry, W. Benz, \& R. Vonsteiger, Space Sci. Ser. of ISSI (Kluwer Academic Publishers), in press

Udry, S., Eggenberger, A., Mayor, M., Mazeh, T., \& Zucker, S. 2003c, in The Environments and Evolution of Double and Multiple Stars, ed. C. Scarfe, \& C. Allen, Rev. Mex. Astron \& Astroph., in press

Vidal-Madjar, A., Lecavelier des Etangs, A., Desert, J.-M., et al. 2003, Nature, 422, 143

Vogt, S., Marcy, G., Butler, R., \& Apps, K. 2000, ApJ, 536, 902

Ward, W. 1997, Icarus, 126, 261

Weidenschilling, S., \& Marzari, F. 1996, Nature, 384, 619

Wuchterl, G. 2000a, in From Extrasolar Planets to Cosmology: The VLT Opening Symp., ed. J. Bergeron, \& A. Renzini, ESO Astrophys. Symp., 408

Wuchterl, G. 2000b, in Planetary Systems in the Universe: Observations, Formation and Evolution, IAU Symp. 202, ed. A. Penny, P. Artymowicz, A.-M. Lagrange, \& S. Russell, ASP Conf. Ser., in press

Zucker, S., \& Mazeh, T. 2002, ApJ, 568, L113 